\title{
Group laws and free subgroups in topological groups
}

\author{
Miklós Abért
}

June 25, 2003

\begin{abstract}
We prove that a permutation group in which different finite sets have different stabilizers cannot satisfy any group law. For locally compact topological groups with this property we show that almost all finite subsets of the group generate free subgroups.

We derive consequences of these theorems on Thompson's group $F$, weakly branch groups, automorphism groups of regular trees and profinite groups with alternating composition factors of unbounded degree.
\end{abstract}

\section{Introduction}

Let $G$ be a permutation group acting on a set $X$. We say that $G$ separates $X$ if for any finite subset $Y \subseteq X$ the pointwise stabilizer $G_{Y}$ does not stabilize any point outside $Y$. A group $G$ satisfies a group law, if there exists a word $w \in F_{k}$ such that $w\left(g_{1}, g_{2}, \ldots, g_{k}\right)=1$ for all $g_{i} \in G$. Our first result is the following.

Theorem 1 If $G$ separates $X$ then $G$ does not satisfy any group law.

In contrast, for the natural transitive permutation action of the so-called lamplighter group $L$ (the wreath product $C_{2}(\mathbb{Z}$ ) the stabilizer of $n$ points stabilizes at most $n$ other points. On the other hand, $L$ is metabelian, so it satisfies a commutator identity.

Theorem 1 allows us to give a short proof of the main result in $[\mathrm{BM}]$. Thompson's group $F$ is the group of piecewise linear orientation-preserving homeomorphisms of the unit interval with dyadic breaking points and slopes which are powers of 2 .

Corollary 2 Thompson's group $F$ does not satisfy any group law.

Note that by $[\mathrm{BM}] F$ does not contain any free subgroups.

We can generalise Theorem 1 for locally compact topological groups which act on a set separating it and show that generic subgroups of them are free. Altough we derive the compact case as a simple consequence of the general result 
on locally compact groups, we state it differently as the necessary restrictions are more natural.

Let $G$ be a compact topological group. Then there is a unique $G$-invariant probability measure $\mu$ on $G$. This extends to a probability measure on $G^{n}$. A subset of $G^{n}$ is almost sure (a.s.) if its complement has zero probability. An action of $G$ on a set $X$ is topological, if for all $x \in X$ the point stabilizer $G_{x}$ is closed. If $G$ is also transitive, then $X$ can be identified with the coset space $G / H$ for any point stabilizer $H$ and as such, it carries a natural $G$-invariant measure $\lambda$.

A group $\Gamma \subseteq \operatorname{Sym}(\Omega)$ acts freely on $\Omega$ if for every $x \in \Omega$ the point-stabilizer $\Gamma_{x}$ is trivial. A group $H \subseteq G$ acts almost freely on $X$ if there is $Y \subseteq X$ such that $\lambda(X \backslash Y)=0, Y$ is $\Gamma$-invariant and $\Gamma$ acts freely on $Y$.

Theorem 3 Let $G$ be a compact topological group acting topologically on $X$ and separating $X$. Then $n$ random elements in $G$ generate a free subgroup of rank $n$ a.s. If $G$ is transitive then this group acts almost freely on $X$ a.s.

So somewhat surprisingly, in the transitive case a generic finitely generated subgroup of $G$ does exactly the opposite of separating $X$, as almost all points have a stabilizer which stabilizes almost all points in $X$.

Theorem 3 applies to profinite weakly branch groups. A weakly branch group can be defined as a group acting spherically transitively on a rooted tree such that the rigid stabilizer of every vertex is nontrivial. Recall that the rigid stabilizer of a vertex $v$ is the set of group elements which move only descendants of $v$. The class of weakly branch groups contains many groups with interesting properties. Examples are the first Grigorchuk group (Gri ), Wilson's group of non-uniform exponential growth (Wi1]), Segal's group of slow subgroup growth ( $\mid \mathrm{Seg})$ and the group constructed by Grigorchuk and Zuk which is not in the class SG (GZ]) but as Bartholdi and Virág have recently shown is amenable ( $\mathrm{BV}]$ ).

A profinite weakly branch group is a weakly branch group which is closed in the profinite topology of the automorphism group of the corresponding tree, e.g., the closure of any weakly branch group.

Corollary 4 If $G$ is a profinite weakly branch group then $n$ random elements generate a free subgroup of rank $n$ in $G$ a.s. Moreover, if the underlying tree has bounded degree and $G$ is finitely generated then $G$ contains a finitely generated dense free subgroup.

The above result generalises a result of Wilson Wi2 who proved that for branch profinite groups the subset of $n$-tuples in $G^{n}$ generating non-free subgroups is meagre (first category) in $G^{n}$. His result implies that finitely generated pro- $p$ branch groups have finitely generated dense free subgroups, but for the general profinite setting our stronger probabilistic result is needed.

Theorem 3 also applies to infinite iterated wreath products of nontrivial finite permutation groups. This reproves a result of Bhattacharjee [Bha] saying that in these groups $n$ elements generate a free subgroup of rank $n$ a.s. 
We deduce Theorem 3 as a special case of a more general theorem on locally compact groups. If the group $G$ is locally compact but not compact, we do not have a $G$-invariant probability measure anymore. Also, we have to strengthen the notion of topological action, since a countable group can easily act on a set, separating it, without containing any free subgroups. Thompson's group or the finitary symmetric group acting on $\mathbb{N}$ both have this property.

A locally compact group $G$ carries a right $G$-invariant Haar measure $\mu$. An action of $G$ on a set $X$ is topological, if for all $x \in X$ the point stabilizer $G_{x}$ is closed and $\mu\left(G_{x}\right)=0$. The action is strongly topological, if for all finite subset $Y \subseteq X$ the pointwise stabilizer $G_{Y}$ acts topologically on $X \backslash Y$. A property of $n$-tuples of $G$ is almost sure (a.s.) if its complement is $\mu$-negligible in $G^{n}$. Note that these notions are independent of the choice of $\mu$. If $G$ is transitive, then $X$ again can be identified with the coset space $G / H$ for some closed subgroup $H$ and as such, it inherits a natural measure $\lambda$ from $G$. This measure is not necessarily $G$-invariant, but always $G$-equivariant.

Theorem 5 Let $G$ be a locally compact topological group acting strongly topologically on $X$ and separating $X$. Then $n$ elements in $G$ generate a free subgroup of rank $n$ a.s. If $G$ is transitive then this group acts almost freely on $X$ a.s.

A natural class of locally compact groups to which Theorem [5 applies are automorphism groups of unrooted regular trees.

Corollary 6 For any regular tree $T, n$ elements in Aut $(T)$ generate a free subgroup of rank $n$ a.s.

This corollary is used in $[\mathrm{AG}$ to show that $A u t(T)$ contains finitely generated dense free subgroups for every unrooted regular tree $T$.

Using an asymptotic version of Theorem 3 we can also answer a question of Pyber. Dixon, Pyber, Seress and Shalev in DPSS gave an elegant new proof to a conjecture of Magnus. The original proof was given by Weigel Weil using different methods. Magnus conjectured that the free group $F_{2}$ is residually $S$ for every infinite set $S$ of pairwise non-isomorphic nonabelian finite simple groups. Recall that a group $G$ is residually $S$ if the intersection of all normal subgroups with quotients in $S$ is trivial in $G$. One of the corollaries in DPSS is that if a profinite group $G$ has infinitely many nonabelian simple quotients then $n$ random elements in $G$ generate a free subgroup a.s. for all $n$.

It is asked in [DPSS, Problem 7] whether one can generalise this to profinite groups with infinitely many nonabelian simple composition factors. Our result makes a first step into this direction.

Theorem 7 Let $G$ be a profinite group which has alternating composition factors of unbounded degree. Then $n$ random elements generate a free subgroup in $G$ a.s. for all n. 


\section{Proofs}

In this section we prove the theorems and corollaries from the introduction.

Proof of Theorem [1. We can assume that $G$ acts transitively on $X$. If the action is intransitive, let $X^{\prime}$ be a nontrivial orbit of $G$. Then $X^{\prime}$ is infinite, otherwise for every $x^{\prime} \in X^{\prime}$ and $Y=X^{\prime} \backslash\left\{x^{\prime}\right\}$ the stabilizer $G_{Y}$ would stabilize $x^{\prime}$, contradicting the separability. By the same argument for every finite $Y \subseteq X$ the orbits of the action of $G_{Y}$ on $X \backslash Y$ are all infinite.

Let $F_{k}$ be the free group on $k$ variables $f_{1}, f_{2}, \ldots, f_{k}$. Let $w \in F_{k}$ be a reduced word of length $n$, i.e.,

$$
w=v_{1} v_{2} \cdots v_{n}
$$

where

$$
v_{i} \in\left\{f_{1}, f_{1}^{-1}, \ldots, f_{k}, f_{k}^{-1}\right\}
$$

with $v_{i} v_{i+1} \neq 1$ for $1 \leq i \leq n-1$.

Let $w_{j}$ be the $j$-th beginning segment of $w$,

$$
w_{j}=v_{1} v_{2} \cdots v_{j} \quad(0 \leq j \leq n) .
$$

For a $k$-tuple $\left(g_{1}, g_{2}, \ldots, g_{k}\right) \in G^{k}$ we denote $w_{j}\left(g_{1}, g_{2}, \ldots, g_{k}\right) \in G$ by the value of the word $w_{j}$ in $G$ via the substitution $f_{i}=g_{i}$.

We claim that for all $1 \neq w \in F_{k}$ and for all $x_{0} \in X$ there exist a $k$-tuple $\left(h_{1}, h_{2}, \ldots, h_{k}\right) \in G^{k}$ such that the points

$$
x_{j}=x_{0}^{w_{j}\left(h_{1}, h_{2}, \ldots, h_{k}\right)} \quad(0 \leq j \leq n)
$$

are disjoint. This clearly shows that $G$ cannot satisfy the group law $w$, as this particular substitution moves the point $x_{0}$ to $x_{n} \neq x_{0}$.

We prove our claim by induction on $n$. For $n=1$ the word consists of one variable and the claim is trivial.

Fix $x_{0} \in X$. Using the inductional hypothesis, we have $\left(g_{1}, g_{2}, \ldots, g_{k}\right) \in G^{k}$ such that $x_{0}, x_{1}, \ldots, x_{n-1}$ are all disjoint. If $x_{n} \notin\left\{x_{0}, x_{1}, \ldots, x_{n-1}\right\}$ then we found the right $\left(g_{1}, g_{2}, \ldots, g_{k}\right)$. So assume that $x_{n}=x_{j}$ for some $j<n$.

Now let

$$
I=\left\{i<n \mid v_{i}=v_{n} \text { or } v_{i+1}=v_{n}^{-1}\right\} .
$$

Let $m$ be the index of $v_{n}$, that is $v_{n} \in\left\{f_{m}, f_{m}^{-1}\right\}$. Also let $g=v_{n}\left(g_{1}, g_{2}, \ldots, g_{k}\right)$ (thus $g$ is either $g_{m}$ or $g_{m}^{-1}$ ). We claim that $j \notin I$. If $v_{j}=v_{n}$, then $x_{j-1}^{g}=$ $x_{j}=x_{n-1}^{g}$ which is a contradiction since $j<n$ and all the $x_{i}$ are disjoint for $i<n$. If $v_{j+1}=v_{n}^{-1}$ then $x_{j+1}^{g}=x_{j}=x_{n-1}^{g}$ which implies $j=n-2$ and thus $v_{n-1}=v_{n}^{-1}$ which contradicts the assumption that the word $w$ is reduced. So our claim holds and $j \notin I$.

Now let $Y=\left\{x_{i} \mid i \in I\right\}$ and let $c \in G_{Y}$ be an arbitrary element of the stabilizer of $Y$. 
Modify the $k$-tuple $\left(g_{1}, g_{2}, \ldots, g_{k}\right)$ to $\left(h_{1}, h_{2}, \ldots, h_{k}\right)$ as follows: $h_{i}=g_{j}$ $(i \neq m), h_{m}=g_{m} c$ if $v_{n}=f_{m}$ and $h_{m}=c g_{m}$ if $v_{n}=f_{m}^{-1}$. Using the construction of $I$ it is straightforward to check that $x_{i}^{h_{i+1}}=x_{i+1}$ for $i<n-1$ and $x_{n-1}^{h_{n}}=x_{j}^{c}$.

Since $x_{j} \notin Y$, the orbit of $G_{Y}$ containing $x_{j}$ is infinite so we can choose $c$ such that $x_{j}^{c} \notin\left\{x_{0}, x_{1}, \ldots, x_{n-1}\right\}$. So the $k$-tuple $\left(h_{1}, h_{2}, \ldots, h_{k}\right)$ shows that our claim holds and so $w\left(g_{1}, g_{2}, \ldots, g_{k}\right) \neq 1$.

Proof of Corollary 2, We show that Thompson's group $F$ separates the unit interval by its natural action. Let $Y \subseteq[0,1]$ be a finite set and let $x \in[0,1] \backslash Y$. Let $d_{1}, d_{2} \in(0,1)$ be dyadic numbers such that $x \in\left[d_{1}, d_{2}\right]$ but $\left[d_{1}, d_{2}\right] \cap Y=\emptyset$. Then the pointwise stabilizer $F_{d_{1}, d_{2}}$ of $\left[0, d_{1}\right] \cup\left[d_{2}, 1\right]$ in $F$ is permutation isomorphic to $F$ by its action on $\left[d_{1}, d_{2}\right]$. In particular, there is an element of $F_{d_{1}, d_{2}}$ which moves the point $x$.

Proof of Theorem 3. We need to check that the conditions for Theorem [5 hold, that is, the action of $G$ on $X$ is strongly topological.

By the same argument as in the proof of Theorem [1 we see that every $G$ orbit has to be infinite. So any point-stabilizer $G_{x}$ must have infinite index in $G$; since $G_{x}$ is closed and $G$ is compact, $G_{x}$ has zero Haar measure, so the action of $G$ is topological. Also, for any finite set $Y \subseteq X$ the pointwise stabilizer $G_{Y}$ trivially separates $X \backslash Y$ and in $X \backslash Y$ the point-stabilizers are closed (being the intersection of closed subgroups). The stabilizers $G_{Y}$ are compact, so by repeating the above argument to the action of $G_{Y}$ on $X \backslash Y$ we see that the action of $G$ on $X$ is strongly topological.

Proof of Corollary 4. Let $G$ act on the rooted tree $T$ spherically transitively such that the rigid vertex stabilizers are nontrivial. Let $X$ be the boundary of the tree, i.e., the set of infinite rays of $T$. Since $G$ is closed in the profinite topology of $A u t(T)$, for every $x \in X$ the stabilizers $G_{x}$ are closed, so $G$ acts on $X$ topologically.

We claim that $G$ separates $X$. Let $y_{1}, \ldots y_{n}, x \in X$ be pairwise disjoint rays. Choose a level $k$ such that the vertices in the rays $y_{1}, \ldots y_{n}, x$ at the $k$-th level are all disjoint. Let $x_{0}$ be the vertex of $x$ at level $k$. Let $S$ be the stabilizer of $x_{0}$ in $G$ and let $R$ be the rigid vertex stabilizer of $x_{0}$ in $G$. Then $S$ acts spherically transitively on the infinite subtree rooted at $x_{0}$, since if $a$ and $b$ are both descendants of $x_{0}$ at the same level, then there is $g \in G$ such that $a^{g}=b$ and clearly $g$ must stabilize $x_{0}$. Now $R$ is normal in $S$ and since it is nontrivial, it cannot stabilize any infinite ray going through $x_{0}$. In particular, there exists $r \in R$ such that $x^{r} \neq x$. On the other hand, $r$ stabilizes every ray not going through $x_{0}$. This proves our claim.

Now using Theorem 3 we see that $n$ random elements generate a free subgroup of $G$ with probability 1 .

If the degree of $T$ is bounded, then $\operatorname{Aut}(T)$ has only finitely many different simple upper composition factors. Thus the degree of upper alternating sections of $G$ is bounded. Recall that an upper section is a homomorphic image of 
an open subgroup. Using BPS this implies that $G$ has polynomial maximal subgroup growth (PMSG), i.e., the number of maximal subgroups of index $n$ in $G$ is bounded by a polynomial of $n$. By MS for the profinite group $G$ PMSG is equivalent to that $G$ is positively finitely generated (PFG), i.e., that there is a number $k$ such that $k$ random elements generate a dense subgroup of $G$ with positive probability.

So for every $n \geq k$ we obtain that $n$ random elements of $G$ generate a dense free subgroup of $G$ with positive probability. This completes the proof.

Now we will prove Theorem [5] We need a straightforward lemma, the proof of which we include for completeness.

If $G$ is a locally compact group and $H$ is a closed subgroup endowed with a right Haar measure $\lambda$, then $\lambda$ naturally extends to every right $H$-coset $C$ by $\lambda(X)=\lambda\left(X g^{-1}\right)$ where $g \in C$. The measure $\lambda(X)$ is independent of the choice of $g$ as $\lambda$ is right invariant in $H$.

Let $K \subset H$ be a closed subgroup of measure zero and let $\mu$ be right Haar measure on $K$.

Lemma 8 Let $X$ be a $\lambda$-measurable subset of $C$ such that for almost all $K$ cosets $D \subseteq C$ we have $\mu(D \cap X)=0$. Then $\lambda(X)=0$.

Proof. Fix $c_{0} \in C$. Then $C=H c_{0}$. Define the subset $k h c_{0}$

$$
Y=\left\{(k, h) \in K \times H \mid k h c_{0} \in X\right\} .
$$

Since $Y$ is the preimage of $X$ by a continuous map from $K \times H$ to $C$, it is measurable. Now for all $h_{0} \in H$ the fiber

$$
R\left(h_{0}\right)=\left\{k \in K \mid\left(k, h_{0}\right) \in Y\right\}=K \cap X c_{0}^{-1} h_{0}^{-1}
$$

and so by the assumption for almost all $h_{0}$ we have

$$
\mu\left(R\left(h_{0}\right)\right)=\mu\left(K h_{0} c_{0} \cap X\right)=0 .
$$

So by Fubini's theorem $Y$ has measure zero in $K \times H$. This implies that for almost all $k_{0} \in K$ the fiber

$$
L\left(k_{0}\right)=\left\{h \in H \mid\left(k_{0}, h\right) \in Y\right\}=k_{0}^{-1} X c_{0}^{-1}
$$

has measure zero in $H$. Pick such a $k_{0}$. Using that $\lambda$ is left $H$-equivariant we get $\lambda\left(X c_{0}^{-1}\right)=0$ in $H$, that is, $\lambda(X)=0$.

Proof of Theorem 5. We will use the notations from the proof of Theorem 1

We claim that we can assume that $G$ is transitive on $X$. Let $X_{0} \subseteq X$ be a $G$-orbit. The restriction $\bar{G}$ of the action of $G$ on $X_{0}$ is a group homomorphism and the kernel $K$ is closed of Haar measure zero since it is the intersection of 
the closed stabilizers of points in $X_{0}$. Now $\bar{G}$ clearly separates $X_{0}$ and it acts strongly topologically on it. Since $K$ has zero measure, the preimage of zero measure subsets of $\bar{G}^{n}$ have zero measure in $G^{n}$ and our claim holds.

Let $Y_{1}, Y_{2}, \ldots, Y_{k} \subseteq X$ and $Z_{1}, Z_{2}, \ldots, Z_{k} \subseteq X$ be finite ordered subsets such that for all $1 \leq i \leq k$ we have $\left|Y_{i}\right|=\left|Z_{i}\right|$ and there exists $h_{i}$ such that $Y_{i}^{h_{i}}=Z_{i}$ as ordered subset. Let $Y=\cup Y_{i}, Z=\cup Z_{i}$ and let

$$
A\left(Y_{1}, Y_{2}, \ldots, Y_{k}, Z_{1}, Z_{2}, \ldots, Z_{k}\right)=\left\{\left(g_{1}, g_{2}, \ldots, g_{k}\right) \mid Y_{i}^{g_{i}}=Z_{i} \text { for } 1 \leq i \leq k\right\} .
$$

Let us denote $S_{i}=G_{Y_{i}}$ the pointwise stabilizer of $Y_{i}$ in $G$, let $C_{i}=S_{i} h_{i}$ and let $S=S_{1} \times S_{2} \times \cdots \times S_{k}$. Obviously, $A$ coincides the right $S$-coset $C_{1} \times C_{2} \times \cdots \times C_{k}$ so it inherits the natural topology and measure from $S$.

Now let $x_{0} \in X \backslash Y$ be a fixed point and let $w \in F_{k}$ be a reduced word of length $n>0$. Call a tuple $\left(g_{1}, g_{2}, \ldots, g_{k}\right) \in A$ distinctive, if for the elements

$$
x_{j}=x_{0}{ }^{w_{j}\left(g_{1}, g_{2}, \ldots, g_{n}\right)}
$$

we have $x_{i} \notin Y \cup Z(1 \leq i \leq n)$ and $x_{i} \neq x_{j}(0 \leq i<j \leq n)$. Let

$$
U=U\left(Y_{1}, Y_{2}, \ldots, Y_{k}, Z_{1}, Z_{2}, \ldots, Z_{k}, x_{0}, w\right)
$$

be the set of distinctive elements of $A$.

Our first claim is that $U$ is open in $A$. Let $H=G_{x_{0}}$ be the stabilizer of $x_{0}$ in $G$. For all $0 \leq i<j \leq k$ let us define

$$
V_{i j}=\left\{\left(g_{1}, g_{2}, \ldots, g_{k}\right) \in A \mid x_{i}=x_{j}\right\} .
$$

Clearly,

$$
U=A \backslash \bigcup_{i<j \leq n} V_{i j}
$$

so it is enough to show that the sets $V_{i j}$ are closed. Define the function $F_{i j}$ : $G^{k} \rightarrow G$ by

$$
F_{i j}\left(g_{1}, \ldots, g_{k}\right)=w_{i}\left(g_{1}, g_{2}, \ldots, g_{n}\right) w_{j}^{-1}\left(g_{1}, g_{2}, \ldots, g_{n}\right) .
$$

Since $F_{i j}$ is continuous, the pre-image $W_{i j}=F_{i j}^{-1}(H)$ is closed in $G^{n}$. On the other hand, $V_{i j}=A \cap W_{i j}$ so it is closed.

Our second claim is that $U$ is a.s. in $A$. We use induction on $n$, the length of $w$. If $n=0$, then $U=A$ and the claim obviously holds.

If $n>0$, let $f$ be the first letter of $w$. By permuting the letters and possibly switching $f_{1}$ and $f_{1}^{-1}$ (and also $Y_{1}$ and $Z_{1}$ ), we can assume that $f=f_{1}$. Now let $R$ be the stabilizer of $x_{0}$ in $S_{1}$ and let $D=R h_{1} \times C_{2} \times \cdots \times C_{k}$. Since $G$ acts strongly topologically on $X, R$ is closed of zero measure in $S_{1}$, thus $D$ is closed of zero measure in $A$. The set

$$
L=\left\{\left(g_{1}, g_{2}, \ldots, g_{k}\right) \in A \mid x_{0}^{g_{1}} \in Y \cup\left\{x_{0}\right\}\right\}
$$


is the finite union of $D$-cosets so it has zero measure in $A$. That is, for almost all $\left(g_{1}, g_{2}, \ldots, g_{k}\right) \in A$ we have $x_{0}^{g_{1}} \notin Y \cup\left\{x_{0}\right\}$. For a fixed $x_{0}^{\prime}=x_{0}^{g_{1}}$ consider the new system

$$
Y_{1}^{\prime}=Y_{1} \cup\left\{x_{0}\right\}, Z_{1}^{\prime}=Z_{1} \cup\left\{x_{0}^{\prime}\right\}
$$

and $Y_{j}^{\prime}=Y_{j}, Z_{j}^{\prime}=Z_{j}$ for $2 \leq j \leq k$. Also let $w^{\prime}=f_{1}^{-1} w$ reduced (delete the first letter of $w)$. Now $w^{\prime}$ has length $n-1$ so by induction $U^{\prime}=U\left(Y_{1}^{\prime}, \ldots, Z_{k}^{\prime}, x_{0}^{\prime}, w^{\prime}\right)$ is a.s. in $A^{\prime}=A\left(Y_{1}^{\prime}, \ldots, Z_{k}^{\prime}\right)$. Since $x_{0} \in Y_{1}^{\prime}$, distinctive elements of $A^{\prime}$ will be also distinctive in $A$. Using Lemma 8 we see that $U$ is a.s. in $A$ what we claimed.

Now we prove the theorem. Let $k \geq 1$, set $X_{1}, \ldots, X_{k+1}$ and $Y_{1} \ldots, Y_{k+1}$ to be all empty sets and let $x_{0} \in X$. For a word $1 \neq v \in F_{k}$ let us define $w \in F_{k+1}$ by $w=f_{k+1} v f_{k+1}^{-1}$. Then using the above claim we get that the set of distinctive $k+1$-tuples is a.s. in $G^{k+1}$. In particular

$$
\left(x_{0}^{g_{k+1}}\right)^{v\left(g_{1}, \ldots, g_{k}\right) g_{k+1}^{-1}}=x_{0}^{w\left(g_{1}, \ldots, g_{k+1}\right)} \neq x_{0}
$$

so

$$
\left(x_{0}^{g_{k+1}}\right)^{v\left(g_{1}, \ldots, g_{k}\right)} \neq x_{0}^{g_{k+1}}
$$

for such $k+1$-tuples. Using Fubini's theorem this implies that for almost all $h \in G$ the set of distinctive $k+1$-tuples with $g_{k+1}=h$ starting from $x_{0}^{h}$ is a.s. in $G^{k}$. So assuming the transitivity of $G$ on $X$ for almost all $x \in X$ the value $v\left(g_{1}, \ldots, g_{k}\right)$ does not fix $x$. Using that there are countably many words in $F_{k}$ and Fubini's theorem again, we get that for almost all $k$-tuples $\left(g_{1}, \ldots, g_{k}\right) \in G^{k}$ for almost all $x \in X$ the value $v\left(g_{1}, \ldots, g_{k}\right)$ does not fix $x$ for all $1 \neq v \in F_{k}$. That is, $\left(g_{1}, \ldots, g_{k}\right) \in G^{k}$ generates a free subgroup acting almost freely on $X$ a.s.

Proof of Corollary [6. Let $X$ be the boundary of the tree, i.e., the set of infinite rays of $T$ and let $G=\operatorname{Aut}(T)$. Then $G$ is locally compact, the neighbourhood of the identity being pointwise stabilizers of balls. We can assume that vertex stabilizers have Haar measure 1. Clearly, for every $x \in X$ the point stabilizer $G_{x}$ is closed. Let $Y \subseteq X$ be an arbitrary (possibly empty) finite subset and let $x \in X \backslash Y$.

If $|Y| \geq 3$, then $G_{Y}$ is compact as it the stabilizer of the convex hull $C$ of $Y$ in $T$. Since $C$ has only finitely many limit points in $X$ (the set $Y$ ), $x$ has a closest point $c$ in $C$. Then $G_{Y}$ acts transitively on the shadow of $c$ containing $x$, so the $G_{Y}$-orbit containing $x$ is infinite (in fact it contains continuously many points). As $G_{Y}$ is compact, this shows that $G_{Y \cup\{x\}}$ has infinite index and thus zero measure in $G_{Y}$.

If $|Y| \leq 2$, then $G_{Y}$ acts transitively on $X \backslash Y$. Fix a point $t \in T$, let $H$ be the stabilizer of $t$ in $G_{Y}$ and let $H_{x}$ be the stabilizer of $x$ in $H$. Then $H$ is compact and has countable index in $G_{Y}$ so it has positive measure in $G_{Y}$. As the convex cone of $Y \cup\{t\}$ has no new boundary points, the $x$-orbit of $H$ is infinite and so $H_{x}$ has zero measure in $H$. Since $G_{Y \cup\{x\}}$ is a countable union of cosets of $H_{x}$, it has zero measure in $G_{Y}$. 
The above argument shows that $G$ separates $X$ and the action is strongly topological. Using Theorem 5 we see that the corollary holds.

Now we prove Theorem 7 using a general lemma on finite permutation groups.

Let $G$ be a permutation group acting on a finite set $X$. We say that $G$ separates $X$ in order $(n, a)$ if for any subset $Y \subseteq X$ with $|Y|=n$ the point stabilizer $G_{Y}$ acts on $X \backslash Y$ in a way such that all orbits have size at least $a$. For a word $w \in F_{k}$ let $P_{G}(w)$ denote the probability that $w$ is not satisfied in $G$.

Lemma 9 Let $G$ be a finite group separating $X$ of order $(n, a)$ and let $w \in F_{k}$ have length $n$. Then $Q(w) \geq\left(1-\frac{n}{a}\right)^{n}$.

Proof. We will use the notations of the proof of Theorem 1

Let $x_{0} \in X$ a fixed point. We estimate $Q(w)$ from below with the probability that $x_{0}, x_{1}, \ldots, x_{n}$ are all disjoint. By induction on $n$, with probability at least $\left(1-\frac{n-1}{a}\right)^{n-1} \geq\left(1-\frac{n}{a}\right)^{n-1}$ the points $x_{0}, x_{1}, \ldots, x_{n-1}$ are all disjoint. Fix such a $x_{0}, x_{1}, \ldots, x_{n-1}$ : by doing that, we are restricting the probability space $G^{k}$ to the direct product of particular cosets of stabilizers in $G$. Now the possible extensions for $x_{n}$ are uniformly chosen from a $G_{Y}$-orbit which does not meet $Y$. Thus this orbit has size at least $a$ and the intersection of this orbit with the set $x_{0}, x_{1}, \ldots, x_{n-1}$ has size at most $n$. So with at least probability $1-\frac{n}{a}$ the randomly chosen $x_{n}$ does not meet $x_{0}, x_{1}, \ldots, x_{n-1}$, proving our lemma.

Proof of Theorem 17. Let $A=A_{k}$ be an alternating upper composition factor of $G$. Using standard technique there exist $m$ and normal subgroups $N \triangleleft M \triangleleft G$ such that $M / N \approx A^{m}$ and $|G: N|$ is finite. Factoring out with the centralizer of $M / N$ in $G$ we get that $G$ quotients to a finite group $\widetilde{G}$ which embeds as a subgroup of the automorphism group of $A^{m}$ which contains $A^{m}$ and acts transitively on the factors.

Now the whole $A u t\left(A^{m}\right)$ is isomorphic to the wreath product $S_{k} \prec S_{m}$ which acts naturally on a set $X$ of $k m$ points. Since the natural action of $A$ on $k$ points separates it of order $(n, k-n)$ for any $n<k-1$, the same holds for the action of $A^{m}$ on $X$. Now $\widetilde{G}$ contains $A^{m}$, so it also separates $X$ of order $(n, k-n)$ for all $n<k-1$.

Now let $w \in F_{k}$ be a word of length $n$. If a word is not satisfied in the quotient group $\widetilde{G}$ then it is not satisfied for any preimage in $G$ and the image of a uniform random element in $G$ is uniform random in $\widetilde{G}$. Using Lemma 9 ] we have

$$
P_{G}(w) \geq P_{\widetilde{G}}(w) \geq\left(1-\frac{n}{k-n}\right)^{n} .
$$

Since $k$ is unbounded, this implies $P_{G}(w)=1$.

Since there are countably many words in $F_{k}$, it follows that with probability 1 random $k$-tuples does not satisfy any word, that is, they generate free subgroups. 
Acknowledgement. The author is grateful to the 'Secret Seminar' for the inspiring milieu and to Laci Pyber, who asked him whether Theorem 7 holds.

\section{References}

[AG] M. Abért, Y. Glasner, Generic subgroups of automorphism groups of trees, preprint

[BV] L. Bartholdi, B. Virág, Amenability via random walks, preprint, http://www.arxiv.org/abs/math.GR/0305262

[Bha] M. Bhattacharjee, The ubiquity of free subgroups in certain inverse limits of groups. J. Algebra 172 (1995), no. 1, 134-146.

[BPS] A. Borovik, L. Pyber, A. Shalev, Maximal subgroups in finite and profinite groups, Trans. Amer. Math. Soc. 348 (1996), no. 9, 3745-3761.

[BM] M. G. Brin and G. C. Squier, Groups of piecewise linear homeomorphisms of the real line, Invent. Math. 79 (1985), no. 3, 485-498.

[DPSS] J. D. Dixon, L. Pyber, A. Seress and A. Shalev, Residual properties of free groups and probabilistic methods, J. Reine Ang. Math. 556 (2003), $159-172$.

[Gri] R. I. Grigorchuk, Just infinite branch groups, in New horizons in pro- $p$ groups, 121-179, Progr. Math., 184, Birkhäuser Boston, Boston, MA, 2000 .

[GZ] R. I. Grigorchuk, A. Zuk, On a torsion free weakly branch group defined by a three state automaton, Internat J. Algebra Comput., 12(1-2), 223246

[MS] A. Mann, A. Shalev, Simple groups, maximal subgroups, and probabilistic aspects of profinite groups, Israel J. Math. 96 (1996), 449-468.

[Seg] D. Segal, The finite images of finitely generated groups, Proc. London Math. Soc. (3) 82 (2001), no. 3, 597-613.

[Wei] T. Weigel, Residual properties of free groups I-III, J. Algebra 160 (1993), no. 1, 16-41., Comm. Algebra 20 (1992), no. 5, 1395-1425., Israel J. Math. 77 (1992), no. 1-2, 65-81.

[Wi1] J. Wilson, On exponential and uniformly exponential growth for groups, preprint, http://www.unige.ch/math/biblio/preprint/2002/growth.ps

[Wi2] J. Wilson, On just infinite abstract and profinite groups, in New horizons in pro- $p$ groups, 181-203, Progr. Math., 184, Birkhäuser Boston, Boston, MA, 2000. 